\title{
ATLAS BASEMAPS IN WEB 2.0 EPOCH
}

\author{
V. Chabaniuk ${ }^{\mathrm{a}}$, O. Dyshlyk ${ }^{\mathrm{b}}$ \\ ${ }^{a}$ Institute of Geography of National Academy of Sciences of Ukraine, Intelligence Systems Geo, Ltd. (ISGeo) - chab3@i.ua, \\ chab@isgeo.kiev.ua \\ ${ }^{\mathrm{b}}$ Institute of Geography of National Academy of Sciences of Ukraine - dyshlyk@ geomatica.kiev.ua
}

KEY WORDS: atlas basemaps, big data, innovative LBS systems and applications, neo-cartography, ubiquitous cartography

\begin{abstract}
:
The authors have analyzed their experience of the production of various Electronic Atlases (EA) and Atlas Information Systems (AtIS) of so-called "classical type". These EA/AtIS have been implemented in the past decade in the Web 1.0 architecture (e.g., National Atlas of Ukraine, Atlas of radioactive contamination of Ukraine, and others). One of the main distinguishing features of these atlases was their static nature - the end user could not change the content of EA/AtIS.

Base maps are very important element of any EA/AtIS. In classical type EA/AtIS they were static datasets, which consisted of two parts: the topographic data of a fixed scale and data of the administrative-territorial division of Ukraine. It is important to note that the technique of topographic data production was based on the use of direct channels of topographic entity observation (such as aerial photography) for the selected scale.
\end{abstract}

Changes in the information technology of the past half-decade are characterized by the advent of the "Web 2.0 epoch". Due to this, in cartography appeared such phenomena as, for example, "neo-cartography" and various mapping platforms like OpenStreetMap. These changes have forced developers of EA/AtIS to use new atlas basemaps. Our approach is described in the article. The phenomenon of neo-cartography and/or Web 2.0 cartography are analysed by authors using previously developed Conceptual framework of EA/AtIS. This framework logically explains the cartographic phenomena relations of three formations: Web 1.0, Web $1.0 \times 1.0$ and Web 2.0 .

Atlas basemaps of the Web 2.0 epoch are integrated information systems. We use several ways to integrate separate atlas basemaps into the information system - by building: weak integrated information system, structured system and meta-system. This integrated information system consists of several basemaps and falls under the definition of "big data". In real projects it is already used the basemaps of three strata: Conceptual, Application and Operational. It is possible to use several variants of the basemap for each stratum. Furthermore, the developed methods of integration allow logically coordinate the application of different types of basemaps into a specific EA/AtIS. For example, such variants of the Conceptual strata basemap as the National map of Ukraine of our production and external resources such as OpenStreetMap are used with the help of meta-system replacement procedures.

The authors propose a Conceptual framework of the basemap, which consists of the Conceptual solutions framework of the basemap and few Application solutions frameworks of the basemap. Conceptual framework is intended to be reused in many projects and significantly reduce the resources. We differentiate Application frameworks for mobile and non-mobile environments. The results of the research are applied in few EA produced in 2014-2015 at the Institute of Geography of the National Academy of Sciences of Ukraine. One of them is the Atlas of emergency situations. It includes elements that work on mobile devices. At its core it is "ubiquitous" subset of the Atlas.

\section{INTRODUCTION}

Basemaps (BM) in the last decade were defined as (Decker, 2000): “... a theme that provides essential information on common land features upon which mapping applications may be performed and from which more specialized data may be derived. Typical base mapping themes include features common to any given region such as transportation, elevation, hydrography, land cover, and boundaries. The broad range of features collected in base maps means that multiple groups can share the same data. There are few set rules on what can be a base map layer or what the scale or the level of detail should be. Determination of these characteristics depends on the needs of the organization developing the data for further use".

During the period from 2000 to 2010 the authors created more than 10 EA/AtIS of so-called "classical type". Developers carried out manually monotonous actions with BM at the creation of everyone such EA/AtIS. Examples of these actions: $\beta \rightarrow \alpha 1)$ selecting from existing sets of topographic maps of scales $1: 500,000,1: 1,000,000,1: 2,500,000$ etc. most suitable and actual; $\beta \rightarrow \alpha 2$ ) making project BM (so-called cartographic bases) by means of some geo-information package (for example, MapInfo Professional); $\alpha \rightarrow \alpha$ ) using project BM for construction of EA/AtIS thematic maps; $\alpha \rightarrow \omega)$ transforming project BM (as a part of developed EA/AtIS) into a format of a final product intended for duplicating on CD/DVD. It is clear that as a result of described actions $\mathrm{BM}$ in final products became independent from initial BM.

In the second half of the last decade, there were changes in information technology which can be characterized as the appearance of "Web 2.0 epoch". Thanks to it, in cartography appeared such phenomena as, for example, "neo-cartography" or "cartographic platform OpenStreetMap (OSM)". The specified changes have compelled developers to reconsider approaches both to BM, and to their atlas "relatives". 
For example, BM of Austria (www.basemap.at, accessed 2016mar-26) is an open Web resource which provides access to the three of four main BM "subsystems": 1) topography, 2) administrative division and addresses, 3) ortho-photo (4th subsystem is cadaster). Scientists of Dutch cartographic school (Kraak, 2009) have proved that the atlas BM should not be considered in a separation from the National Spatial Data Infrastructure (NSDI). Moreover, in the constructed prototype of the National atlas of the Netherlands described above actions $\beta \rightarrow \alpha, \alpha \rightarrow \alpha, \alpha \rightarrow \omega$ were dynamic or automated.

Simplistically the atlas BM (ABM) can be defined as BM, applied to the decision of any task in some EA/AtIS. Because such tasks are a few, it is necessary to deal with set of ABM. In modern conditions these ABM cannot be considered independently. Therefore, it is necessary to define $\mathrm{ABM}$ also as an element of some ABM system. This system, at least, should support research and/or creation and/or operation/support and/or development/modernization of EA/AtIS.

The main motivations for the creation of described below ABM Conceptual framework are:

1. M1. The need for creation the new modern EA and AtIS Their ABM should consider existed in the country BM.

2. M2. The need to use the popular mobile devices.

3. M3. The need to react on such initiatives, as INSPIRE (ELF) and NSDI.

4. M4. The need to respond to the rapid development of Web 2.0 cartography, public geo-platform appearance (such as OSM and Google Maps), as well as the variability geoplatform services, including their disappearance.

5. M5. The risk of partial or total loss of working capacity of EA and AtIS created in last decade.

The terms "pattern" and "framework", often used below, are understood as follows. The pattern is a proven best-practice solution to a known, recurring problem within a given context. The architectural pattern is a general, reusable solution to a commonly occurring problem in architecture of Cartographic system within a given context. Framework is architectural pattern for whole Cartographic system (or System of maps, or AtIS, or EA) or some its logical parts.

\section{MAIN BODY}

\subsection{Neocartography and Web 2.0 cartography}

In the work (Chabanyuk, Dyshlyk, 2014) the Conceptual framework ( $\mathrm{CoFr})$ of the National Atlas of Ukraine (NAU) was received by abductive reasoning. This framework is valid for all EA and AtIS of classical type. It can be designated as Classical AtIS CoFr. In view of the Web 1.0 technologies used in the implementation of the NAU, also it can be designated as Web 1.0 AtIS CoFr. Concepts of Classical EA/AtIS and/or Web 1.0 are explained by Figure 1.

Notes to Figure 1:

a) Communication of Cartographic Information (Kolachny, 1977): $U_{1}$ - reality (the universe) represented as seen by the cartographer; L - cartographic language as a system of map symbols and rules for their use; $\mathrm{S}_{1}$ - the subject representing reality, i.e., the cartographer; $\mathrm{M}$ - the product of cartography, i.e., the map; $S_{2}$ - the subject consuming the map, i.e., the map user; $\mathrm{U}_{2}$ - reality (the universe) as seen by the map user; and $\mathrm{I}_{\mathrm{c}}$ - cartographic information.
The creation and communication of cartographic information is actually a very complex process of activities and operations with feedback circuits on various levels. The dynamics of this process are simplified to 7 basic stages in the graph, stages 1 to 4 representing the creation of the map, and stages 5 to 7 its consumption.

b) Representation is taken from (accessed 2016-mar-06) http://www.consultantebranchee.com/2013/01/21/chroniqu e-branchee-no-3-le-web-1-0-2-0-3-0-et-reactif-go/. Producteur - producer, internautes - internet users.

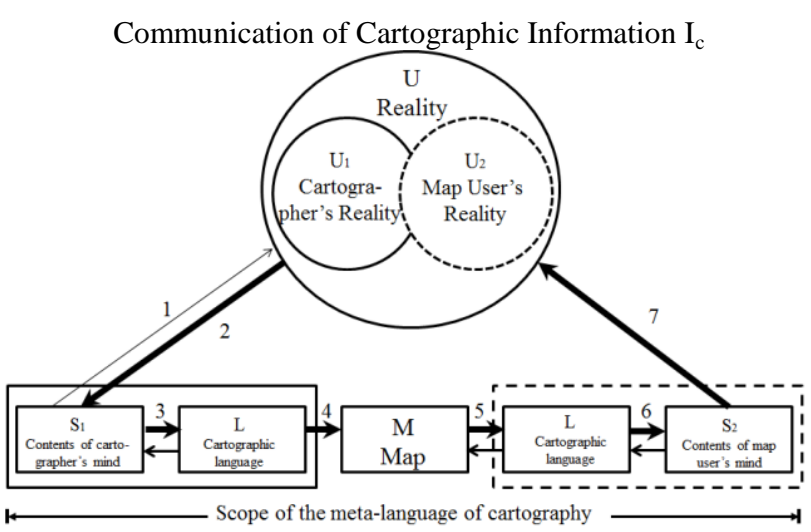

a)

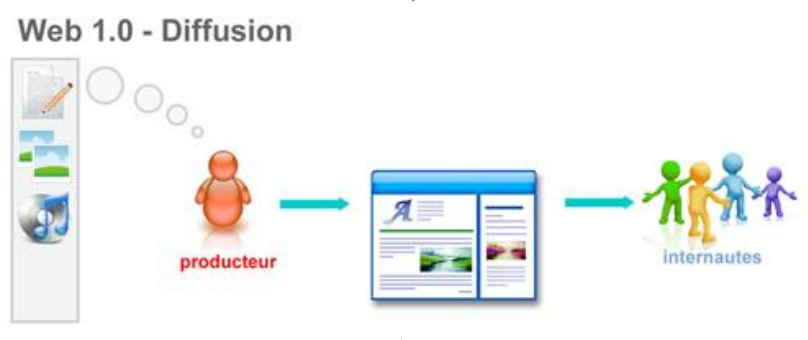

b)

Figure 1. Concepts in EA/AtIS CoFr: (a) - «classical type», (b) «Web 1.0»

Pay attention to the following characteristics of Web 1.0 EA/AtIS CoFr:

1. Usage of the Web 1.0 technologies for construction of classical type EA/AtIS is not restriction. It can be prove that all classical type EA/AtIS can be realized by means of the Web 1.0 technologies. As an example - all of ours EA and AtIS of the last decade, including NAU, are created by means of the Web 1.0 technologies.

2. The Web 1.0 is also called Read Web or Diffusion Web. It means that the content can change only Producer (Cartographer). Internet user (Map User) can only "read" content. In this sense, the Web 1.0 is called as a static Web.

Web 1.0 EA/AtIS CoFr in work (Chabanyuk, Dyshlyk, 2015) was extended to the Web 1.0x1.0 and the Web 2.0 through an generalization and extrapolation of its elements and architecture. The "full" Conceptual framework, received as a result, defines the structure EA/AtIS both classical and neoclassical (or non-classical) types. The term "neoclassical/non-classical" was introduced to describe EA/AtIS which "are beyond" the definition of classical type EA/AtIS. There are variety of the cartographic phenomena «behind boundaries» actually classical cartographies. These cartographic phenomena are designated by many terms with not clearly defined meaning. Partial list of these terms: neogeography, neo-cartography, GeoWeb, GeoSpatial Web, 
Volunteered Geographic Information, crowdsourcing, geo mash-ups, geostack. Let's consider, for example, the term «neogeography».

In the brochure that has already become a classic (Turner A., 2006) neo-geography is defined as: "Neo-geography means 'new geography' and consists of a set of techniques and tools that fall outside the realm of traditional GIS, Geographic Information Systems. Where historically a professional cartographer might use ArcGIS, talk of Mercator versus Mollweide projections, and resolve land area disputes, a neogeographer uses a mapping API like Google Maps, talks about GPX versus KML, and geotags his photos to make a map of his summer vacation. Essentially, Neo-geography is about people using and creating their own maps, on their own terms and by combining elements of an existing toolset. Neo-geography is about sharing location information with friends and visitors, helping shape context, and conveying understanding through knowledge of place. Lastly, neo-geography is fun ..."

Apparently, the most important part of this definition is the last phrase: "Lastly, neo-geography is fun ...". The previous phrases look disputable enough. Let's take phrase "Neo-geography means 'new geography' and consists of a set of techniques and tools ...". The term 'geography' refers to the science with a long history. The adjective 'new' should not change sense of the basic term; otherwise it is not geography. Besides, the geography cannot be reduced to the set of techniques and tools here 'traditional GIS'. Further the geography is actually replaced by the cartography, and the geographer - by the cartographer: "the professional cartographer can use ArcGIS ...", and "the neo-geographer uses cartographical API ...".

The indirect definition from (Haklay, et al., 2008) is more correct: "Central to Web Mapping 2.0 is the concept of neogeography. The term is attributed to Di-Ann Eisnor (2006) of Platial.com - 'a socially networked mapping platform which makes it easy to find, create, share, and publish maps and places' and the essence of neo-geography according to Turner". This definition is agreed upon with the definition from (O'Reilly, 2006): "Web 2.0 is the business revolution in the computer industry caused by the move to the internet as platform, and an attempt to understand the rules for success on that new platform. Chief among those rules is this: Build applications that harness network effects to get better the more people use them. (This is what I've elsewhere called 'harnessing collective intelligence.')"

Pay attention that the key terms/concepts here are "platform" and "collective intelligence". These concepts are the most important distinctive characteristics of the Web 2.0. That is why the emergence of collectively used (geo)platforms can be argued about the beginning of a new epoch as the Internet, and cartography. Notice, that "a platform is a system that can be reprogrammed and therefore customized by outside developers - users - and in that way, adapted to countless needs and niches that the platform's original developers could not have possibly contemplated, much less had time to accommodate" (Andreessen, 2007).

"Neo-cartography" is newer term in comparison with neo-geography. However, professional cartographers avoid defining this term directly. For example, in the article dedicated to neo-cartography (Kraak, 2011) term "neo-cartography" is not defined at all and term "neo-geography" is defined as: "All activities that combine Web 2.0 and maps are also known as neo-geography". (Cartwright, 2012) gives the following indirect definition of neo-cartography: "Relatively recently, maps have been published on the Web by user/producers using a process called 'mashups' with Web 2.0 and Social Software. Web 2.0 is the use of the Web by individuals and groups of individuals to provide and share information, including geographical information. It provides a new model for collaborating and publishing. Users are able to develop their own 'marked-up' maps by appending their overlay information as an additional layer of information, usually using the default symbology provided (and usually map pins are employed), to self-publish their maps via the Web. This has been given many names, including 'Neo-cartography'.”

Representations of the Web 2.0 (Read/Write Web, Collaboration Web) and the Web 3.0 (Semantic Web, Web of Data) are shown in Figure 2.

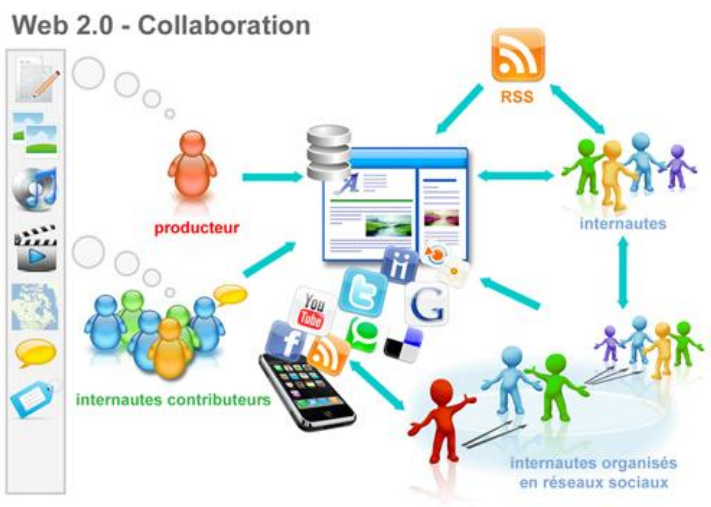

a)

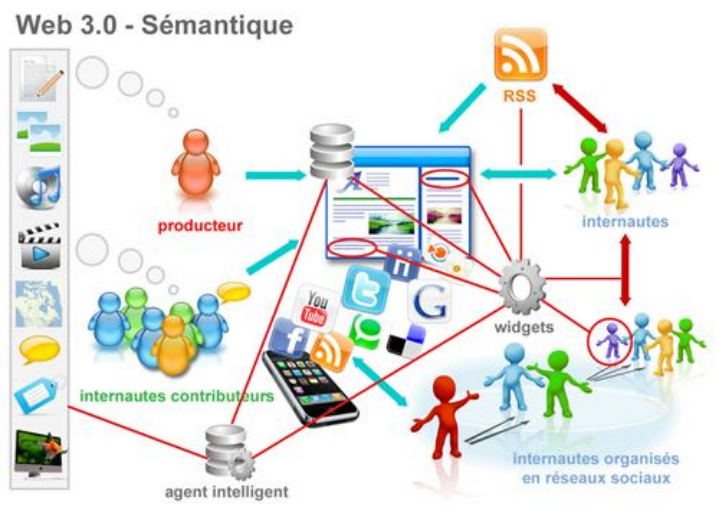

b)

Figure 2. Visual comparison of Web 2.0 and Web 3.0

Notes to Figure 2: Producteur - producer, internautes - internet users, contributeurs - contributors, reseaux sociaux - social networks, organise - organised, agent intelligent - intelligent agent. Representations are taken from (accessed 2016-mar-06) http://www.consultantebranchee.com/2013/01/21/chroniquebranchee-no-3-le-web-1-0-2-0-3-0-et-reactif-go/.

As appears from Figure 2, the Web 2.0 and the following Web 3.0 are characterized by appearance of set of relations between the various elements of the specified system phenomena. These relations are realized by means of various platforms. Therefore, in the Web 2.0+ epoch it is necessary to deal not with separate maps, but with systems in which are used the set of maps, cartographical platforms and relations between them. 


\subsection{Systematics of the atlas basemaps of Web 2.0}

In this section we consider Web $2.0 \mathrm{ABM}$ systematics ${ }^{1}$, of which follows that in the Web 2.0 epoch, it is necessary to deal not with separate ABM, but with some of their system. This system should be built according to certain backbone principles or systematization of ABM.

We allocate the following backbone ABM principles:

1. As EA/AtIS, and used in them ABM are understood in some broader sense. It means that we deal both with final products, and with some «expanding elements». Final products refer to the operation life cycle phase of some EA/AtIS. Expanding elements refer to life cycle phases of research, creation, support and development/modernization of that EA/AtIS. Final products, expanding elements and the relations between them are forming the information system in the broader sense (ISb). Phases of research, creation and operation correspond with so-called Conceptual, Applied and Operational strata accordingly.

2. In practice each ISb is realized as the integrated system of the elements existing on listed above life cycle phases of some EA/AtIS. Integration of elements of such system is carried out by ways which can be ordered on a scale "weak-strong" integration. The realization of "connected to" relation by means of hyperlinks between system elements is the elementary variant of «weak element» integration. More "strong element» integration relations are "depends on", "is part of", "made", "refers to", "uses", "is an example of", and so on. It is known (Hart, Dolbear, 2013) that the listed relations reflect evolution the Web from the Web 1.0 through the Web 2.0 to the Web 3.0. Strong integrated Web 3.0 systems can also be called semantically integrated systems. The ABM system is also evolving. In this work, we investigate Web 2.0 ABM system which includes ABM systems of previous "formations": Web 1.0 and Web 1.0x1.0.

3. Weak- and even semantically integrated ISb is difficult operated because of a considerable quantity of elements between which many relations are constructed. Therefore, in practice integration between system components is (still) applied. The components are groups of system elements and their relations, united on this or that principle. Subsystems are an important and practically useful kind of components. Subsystems are integrated into system by two well-known ways: 1) construction of structured system and 2) construction of meta-system.

4. The last backbone principle is search and application of patterns. Thus we emphasize so-called relational patterns. The essence of relational patterns is repeated relations between elements and components of studied system.

The systematics of Web 2.0 ABM will be explained by the examples from the Atlas of Emergency Situations (ES) of Ukraine (AtlasES). The project was executed within 2010-2015 in the Institute of Geography of the National Academy of Sciences of Ukraine. Two versions of this EA - AtlasES1 and AtlasES2 - were anticipated to be created according to the concept of the AtlasES in the 2010. AtlasES1 had to be the atlas of classical type and should be manufactured by means of Web 1.0 Atlas Solutions Framework (AtlasSF1.0). AtlasSF1.0 was

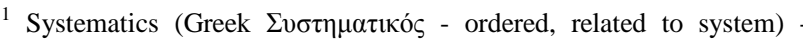
bringing in system, and also the system classification of the studying subject. Often systematics is an auxiliary discipline that helps to order objects, which studies this science; for example, language systematics (https://uk.wikipedia.org/wiki/Систематика, accessed 2016-Mar-20).
}

used for the creation of National Atlas of Ukraine (NAU) and many others classical EA and/or AtIS over the 1999 to 2010 period. AtlasES2 had to be the atlas of geo mash-up type and could be manufactured in the case of some favorable conditions.

AtlasES1 was developed to the full extent in 2010-2014. 142 maps of AtlasES1 were structured into 5 divisions: introduction; preconditions of the ES potential sources; dangers of possible worsening of the living conditions of the citizen and the work of enterprises; ES in Ukraine; prevention of ES. AtlasES2 is developed in 2015 for the subset of AtlasES1 and is called the Atlas of potentially dangerous objects.

Several important events happened in the IT industry during the execution of the AtlasES project. It resulted in the emergence of danger of the loss of functions of AtlasSF1.0 and the manufactured with its application final products. That is why AtlasES1 was issued in two variants: AtlasES1.0 and AtlasES1.0+. AtlasES1.0 and AtlasES1.0+ differ in AtlasSF technologies. AtlasSF1.0+, contrary to AtlasSF1.0, was built on the modern technological triad HTML5+CSS3+JavaScript. This fact is designated by the sign «+». AtlasSF1.0+ ensured the creation of EA/AtIS of the classical type.

The architecture of AtlasES in a broader sense is shown on the Figure 3. It includes the elements of EA, which are not "visible" for the end user.

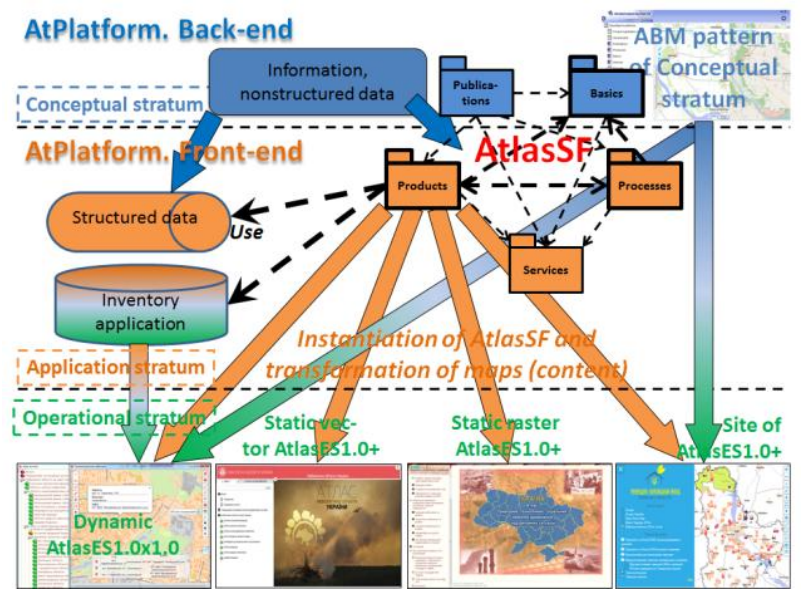

a) Architecture of the "broader" AtlasES

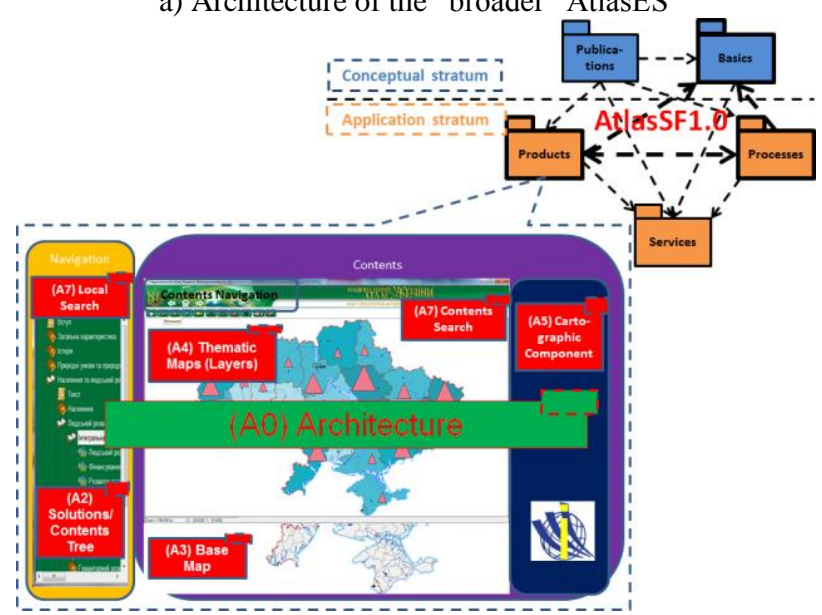

b) Product package of Atlas $\mathrm{S}$ 1.0 (NAU example) Figure 3

1. AtlasES is built on the so-called Atlas platform (AtPlatform, AP), which consists of Back-end and Front-end. AP Back-end consists of the elements of the Conceptual stra- 
tum (blue color) of the full EA/AtIS CoFr (not all elements are shown). The important element of AP Back-end is the shown on the Figure $3 \mathrm{a}$ ABM pattern of the Conceptual stratum. AP Front-end consists of the elements of Application stratum (orange color) of the full EA/AtIS CoFr. The important element of the AP Front-end is the shown on the Figure $3 \mathrm{~b}$ - AtlasSF (part). AtlasSF includes several ABM patterns of the Application stratum. AP Front-end and the included in it AtlasSF of the version 2015 allow constructing several end user (final) products. These products refer to Operational stratum (green color) of the EA/AtIS CoFr (see Figure 3a).

2. The arrows with the changing color show the dynamic relations between elements of the corresponding stratum. Not black (color) arrows with unchanging color mean that the element of lower stratum exists on the highest stratum, but is used on the lower one.

3. Own variants of ABM exist on the Operational stratum in every final product. Several ABM are used in the broader AtlasES on every stratum: Conceptual, Application and Operational.

4. AtlasES1.0 (is not shown on the Figure 3a) was built with the usage of AtlasSF1.0. AtlasES1.0+ (on the Figure 3a is shown as Static raster AtlasES1.0+) - with the usage of AtlasSF1.0+. Product package of the AtlasSF1.0 is shown on the Figure 3b. Notes to the Figure 3b: 1) (A3) Base Map is «product part» of the ABM pattern of the Web 1.0 Application stratum; 2) not shown patterns: (A1) User interface, (A8) Presentation.

Based on Figure 3b several ABM, which are in some relations between each other, exist in the broader AtlasES. That is some $\mathrm{ABM}$ information system is included in the broader AtlasES as subsystem. For initial, the most general determination of this system, we use the notion of information system in the broader sense (ISb): "The totality of all formal and informal data representation and processing activity within an organization, including the associated communication, both internally and with the outside world" (Falkenberg, Lindgreen, 1987). The organization is also understood here in the broader sense - it is the whole so-called Usage World or Organizational level in the EA/AtIS CoFr (Chabanyuk, Dyshlyk, 2015). The determination of the ABM system as ISb is necessary from theoretical and practical points of view:

- Theoretical. Information systems theory is well developed. Thanks to this approach its achievements can be applied to the ABM systems.

- Practical. In the IT industry there are means for ISb realization - portals. The problem of support of constantly updated $\mathrm{ABM}$ can be solved by the right choice of portal.

Full EA/AtIS CoFr structure can be presented in threedimensional nonmetric space: Levels, Strata and Formations. Using the Formations-Strata projection of EA/AtIS CoFr, we obtained the analogue projection of the Web 2.0 ABM system.

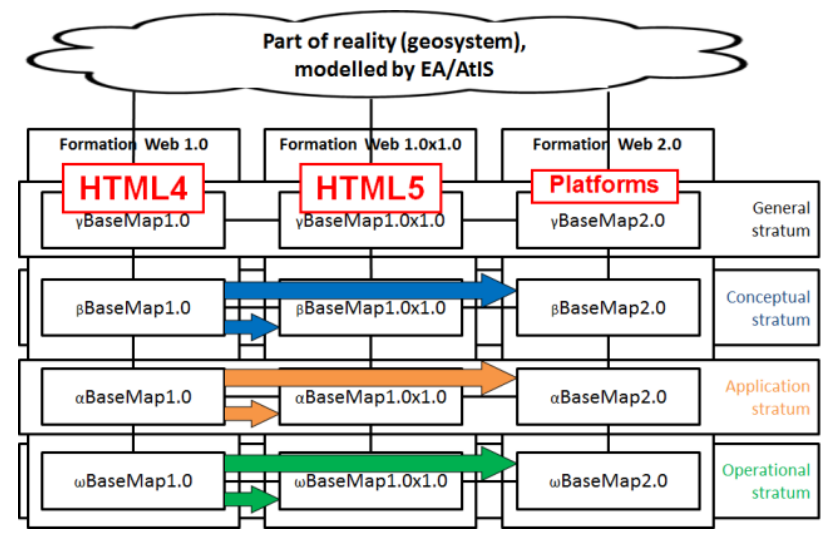

Figure 4. Formation-Strata projection of Web 2.0 ABM System

Notes to Figure 4:

1. Greek letters $\omega, \alpha, \beta, \gamma$ identify the elements of Operational, Application, Conceptual and General strata.

2. All the shown elements of Application and Conceptual strata (for example, $\beta$ BaseMap $1.0^{2}$ ) are the Solution frameworks. It means that they are formed by the elements and corresponding relations from 5 packages: Products, Processes, Basics, Services, Publications (see for example Figure $3 \mathrm{~b}$ ). The example of product is the topographic map (data file and the information description). «Materialized» example of the process is the manual that describes the process of ABM usage during thematic map construction. Every element of Products, Processes and Publications has the corresponding meta-element in the Basics package. The elements of Publications and Basics packages are on the higher stratum than the corresponding to them elements of Products and Processes packages. The Services package fulfills the service functions.

3. The arrows show the directions of generalizations and extrapolations that are done for every 8 elements-patterns (A1-A8) and architecture (A0) from AtlasSF1.0 (Figure 3b) during obtainment of full EA/AtIS CoFr. For example, to obtain $\beta$ BaseMap1.0 $0^{2}$ we used as an initial point $\beta$ BaseMap1.0. Further we took into consideration the needs of $\beta$ BaseMap $1.0^{2}, \quad \alpha$ BaseMap $1.0^{2}$, and also «influence» of particular features of higher strata and formations: HTML5 and (Geo)Platforms (are shown in red).

In the past decade during predominance of the EA/AtIS Web 1.0 Formation, ABM was also described as ISb. The peculiarity of this system was weak integration of its elements. It means in particular that the relation between $\mathrm{ABM}$ of different strata were manual. In practice the weak integration of ISb was realized using hyperlinks. The relations of ABM Web 1.0x1.0 and 2.0 Formations are stronger. Full systematics of Web 2.0 $\mathrm{ABM}$ is shown on the Figure 5. 


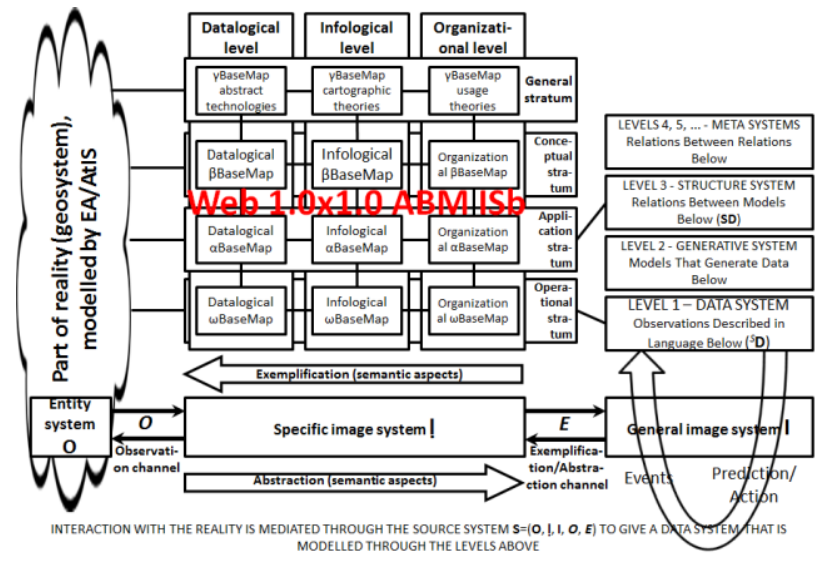

Figure 5. Web $1.0^{2}$ Formation part of full Web 2.0 ABM systematics. Levels-Strata projection is shown

Notes to Figure 5:

1. Figure 5 was obtained by addition to the Figure 4 of the two constructions from (Klir, 1985): "Figure 1.3 Hierarchy of epistemological levels of systems: a simplified overview" (on the Figure 5 it is the right part) and "Figure 2.3 Conceptual elements involved in defining a source system on an object" (on the Figure 5 it is the lower part that in the Klir's Figure 1.3 is called the Level 0 - Source system).

2. Source system $\mathbf{S}$, data system with the semantics ${ }^{S} \mathbf{D}$ and structure data system $\mathbf{S D}$ are described below. $\mathbf{S}$ belongs to the EA/AtIS CoFr General stratum, but here we showed it as in the (Klir, 1985).

Figures 4 and 5 represent different projections of the threedimensional Web 2.0 ABM CoFr. In order to investigate the system properties of this $\mathrm{CoFr}$ we built the system model (SM) with the usage of the approach from (Klir, 1985). SM allows determining the integration methods of different $\mathrm{ABM}$ in integrated hierarchical system more exactly. We used two methods: structure system and meta-system. The shorten fragment of structure system SD is described below.

$\mathrm{SM}$ of the Web 2.0 ABM CoFr could be the data system with semantics ${ }^{S} \mathbf{D}$, represented below:

${ }^{S} \mathbf{D}=(\mathbf{S}, \mathrm{d})$, where

$\mathbf{S}=(\mathbf{O}, \underline{1}, \mathbf{I}, \boldsymbol{O}, \boldsymbol{E})-$ source system,

$\mathrm{d}: \mathbf{W} \rightarrow \mathbf{V}$ - data function, where

$\left.\mathbf{O}=\left(\left\{\mathrm{a}_{\mathrm{i}}, \mathrm{A}_{\mathrm{i}}\right) \mid \mathrm{i}=\{1, \ldots, 11\}\right\}, \quad\left\{\left(\mathrm{b}_{\mathrm{j}}, \mathrm{B}_{\mathrm{j}}\right) \mid \mathrm{j}=\{1,2,3\}\right\}\right)$ - entity system, where

$a_{i}$ - property and $A_{i}$ - set of its appearances, $b_{j}-$ backdrop and $\mathrm{B}_{\mathrm{j}}$ - set of its elements; $\mathbf{W}=\mathrm{W}_{1} \mathrm{xW}_{2} \mathrm{xW}_{3}, \mathbf{V}=\mathrm{V}_{1} \mathrm{xV}_{2} \mathrm{x} \ldots \mathrm{xV}_{11}, \mathrm{~W}_{\mathrm{j}}$, $\mathrm{j}=\{1,2,3\}, \mathrm{V}_{\mathrm{i}}, \mathrm{i}=\{1, \ldots, 11\}$, are determined further.

\begin{tabular}{|l|l|}
\hline Property & Value \\
\hline $\begin{array}{l}\mathrm{a}_{1} \text { : Datum, Control } \\
\text { data and Monuments } \\
\text { (TMC) }\end{array}$ & $\begin{array}{l}\text { Points of state geodetic network, } \\
\text { principal points, points of leveling } \\
\text { network, etc. }\end{array}$ \\
\hline $\mathrm{a}_{2}:$ Relief (TMC) & Contours, reduction, fill, etc. \\
\hline$\ldots$ & $\ldots$ \\
\hline $\begin{array}{l}\mathrm{a}_{7}: \text { Land cover and } \\
\text { soil (TMC) }\end{array}$ & Land cover, soil \\
\hline $\mathrm{a}_{8}:$ Boundaries & $\begin{array}{l}\text { Include village, city (municipal), } \\
\text { regional, district, national boundaries. } \\
\text { Very often the boundaries show } \\
\text { specialized landownership (parks, } \\
\text { airports, military facilities and wildlife }\end{array}$ \\
\hline
\end{tabular}

\begin{tabular}{|l|l|}
\hline Property & Value \\
\hline & reserves) \\
\hline $\begin{array}{l}\mathrm{a}_{9}: \text { Administrative } \\
\text { territorial division }\end{array}$ & $\begin{array}{l}\text { Administrative-territorial division of } \\
\text { Ukraine }\end{array}$ \\
\hline $\begin{array}{l}\mathrm{a}_{10}: \text { Cadastral } \\
\text { information }\end{array}$ & Landownership and land plot borders \\
\hline $\mathrm{a}_{11}:$ Digital images & Digital aero- and space ortho-photos \\
\hline
\end{tabular}

Table 1 . Values of properties $a_{i}$. (TMC) means that the property is determined according to the column «Classification group» of TopoMaps Classifier, used in Ukraine

\begin{tabular}{|l|l|}
\hline Backdrop & Value \\
\hline $\mathrm{b}_{1}:$ Time & $\begin{array}{l}\text { Time period during which the base map of } \\
\text { Ukraine exists. Similar record is t. }\end{array}$ \\
\hline $\mathrm{b}_{2,3}:$ Surface & $\begin{array}{l}\text { Combination of Earth surfaces within the } \\
\text { borders of Ukraine in different periods of its } \\
\text { existence. Similar record is }(\mathrm{x}, \mathrm{y}) .\end{array}$ \\
\hline
\end{tabular}

Table 2. Values of backdrops $b_{j}$

Specific image system

$\mathrm{l}=\left(\left\{\left(\mathrm{v}_{\mathrm{i}}, \mathrm{V}_{\mathrm{i}}\right) \mid \mathrm{i}=\{1, \ldots, 11\}\right\},\left\{\left(\mathrm{w}_{\mathrm{j}}, \mathrm{W}_{\mathrm{j}}\right) \mid \mathrm{j}=\{1,2,3\}\right\}\right)$

General image system

$\mathbf{I}=\left(\left\{\left(\mathrm{v}_{\mathrm{i}}, \mathrm{V}_{\mathrm{i}}\right) \mid \mathrm{i}=\{1, \ldots, 11\}\right\},\left\{\left(\mathrm{w}_{\mathrm{j}}, \mathrm{W}_{\mathrm{j}}\right) \mid \mathrm{j}=\{1,2,3\}\right\}\right)$

Observation channel

$\boldsymbol{O}=\left(\left\{\left(\mathrm{A}_{\mathrm{i}}, \mathrm{V}_{\mathrm{i}}, \mathrm{o}_{\mathrm{i}}\right) \mid \mathrm{i}=\{1, \ldots, 11\}\right\},\left\{\left(\mathrm{B}_{\mathrm{j}}, \mathrm{W}_{\mathrm{j}}, \omega_{\mathrm{j}}\right) \mid \mathrm{j}=\{1,2,3\}\right\}\right)$, where $o_{\mathrm{i}}: A_{\mathrm{i}} \rightarrow V_{\mathrm{i}}, \omega_{\mathrm{j}}: \mathrm{B}_{\mathrm{j}} \rightarrow W_{\mathrm{j}}$.

Abstraction/Exemplification channel

$\left.\boldsymbol{E}=\left(\left\{\left(\mathrm{V}_{\mathrm{i}}, \mathrm{V}_{\mathrm{i}}, \mathrm{e}_{\mathrm{i}}\right) \mid \mathrm{i}=\{1, \ldots, 11\}\right\},\left\{\mathrm{W}_{\mathrm{j}}, \mathrm{W}_{\mathrm{j}}, \varepsilon_{\mathrm{j}}\right) \mid \mathrm{j}=\{1,2,3\}\right\}\right)$, where $\mathrm{e}_{\mathrm{i}}: \mathrm{V}_{\mathrm{i}} \rightarrow \mathrm{V}_{\mathrm{i}}, \varepsilon_{\mathrm{j}}: \mathrm{W}_{\mathrm{j}} \rightarrow \mathrm{W}_{\mathrm{j}}$.

Inversed regarding $e_{i}$ and $\varepsilon_{j}$ functions define abstraction correspondingly $\mathrm{v}_{\mathrm{i}}$ and $\mathrm{w}_{\mathrm{j}}: \mathrm{e}_{\mathrm{i}}^{-1}: \mathrm{V}_{\mathrm{i}} \rightarrow \mathrm{V}_{\mathrm{i}}, \varepsilon_{\mathrm{j}}^{-1}: \mathrm{W}_{\mathrm{j}} \rightarrow \mathrm{W}_{\mathrm{j}}$.

Unfortunately the represented model is too idealized. It is well known that no organization in Ukraine can obtain all necessary values of specific variables $\underline{v}_{i}$ and parameters $w_{j}$ by means of observations or measurements. That is why it is necessary to use the structure system method thanks to which the complete system can be obtained from separate systems or subsystems. In this case every constituent data system is built separately and then it is integrated into the complete system SD.

$\mathbf{S D}=\left\{\left({ }^{\mathrm{m}} \mathrm{V},{ }^{\mathrm{m}} \mathbf{D}\right) \mid \mathrm{m}=\{1,2,3,4\}\right\}$, where

${ }^{1} \mathrm{~V}=\mathrm{V}_{1} \mathrm{x} \ldots \mathrm{x} \mathrm{V}_{8}, \mathrm{~V}_{\mathrm{j}}, \mathrm{j}=\{1, \ldots, 8\}$ is the same as in (6), ${ }^{1} \mathbf{D}-$ corresponding ${ }^{1} \mathrm{~V}$ data system of the topographic map of Ukraine;

${ }^{2} \mathrm{~V}=\mathrm{V}_{8} \mathrm{x} \mathrm{V}_{9}, \mathrm{~V}_{8}, \mathrm{~V}_{9}$ is the same as in (6), ${ }^{2} \mathbf{D}$ - corresponding ${ }^{2} \mathrm{~V}$ data system of the map of the administrative-territorial division of Ukraine;

${ }^{3} \mathrm{~V}=\mathrm{V}_{8} \mathrm{x} \mathrm{V}_{10}, \mathrm{~V}_{8}, \mathrm{~V}_{10}$ is the same as in (6), ${ }^{3} \mathrm{D}$ - corresponding ${ }^{3} \mathrm{~V}$ data system of cadastral index map of Ukraine;

${ }^{4} \mathrm{~V}=\mathrm{V}_{8} \mathrm{xV}_{11}, \mathrm{~V}_{8}, \mathrm{~V}_{11}$ is the same as in (6), ${ }^{4} \mathbf{D}$ - corresponding ${ }^{4} \mathrm{~V}$ data system of ortho-photo map of Ukraine.

\subsection{Web 2.0 ABM CoFr as ABM investigation tool}

Formation Web $1.0 \times 1.0$ or Web $1.0^{2}$ was introduced by us for more adequate description of non-static EA/AtIS of the classical type. Here we mean that in static EA/AtIS of the classical type the changes can be made only on the Application stratum, moreover, by the professional cartographers/cyberneticians. Broadly speaking the end users can only visualize (read) atlas content. Such atlases are closer to the view-only than to the interactive atlases that are defined in (Kraak, Ormeling, 2010).

(Cauvin, et al., 2010) indicate that: "Electronic atlases can be classified according to several principles, particularly their spe- 
cific spatio-thematic features (content of the atlas and space concerned), the use and users envisaged, and lastly, the technical characteristics ... With the first criterion essentially being linked to the thematic domain, a fundamental classification to take into account concerns the uses, users or more precisely, the degree of freedom accorded to them. All the authors are in agreement as to distinguishing three types of atlas according to the levels of freedom, and therefore of interactivity: view-only atlases (or read-only atlases), interactive atlases and analytical atlases."

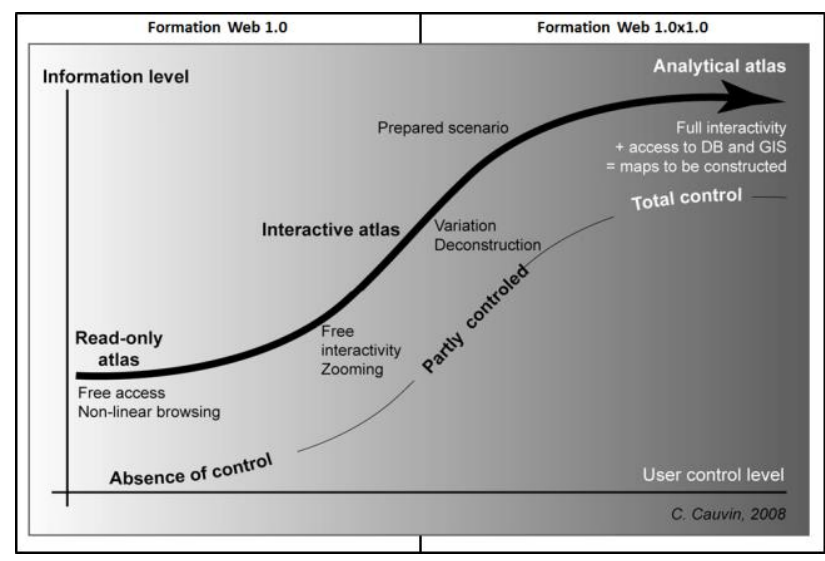

Figure 6. From the view-only atlases to the analytical atlases (Cauvin, et al., 2010; Figure 3.3).

Atlas classes from (Cauvin, et al., 2010; Figure 3.3) are projected on the Web 1.0 and Web 1.0x1.0 Formations. Nonstatic EA/AtIS of the classical type are called also dynamic or mobile EA/AtIS of the classical type. On the Figure 3a is shown one such atlas - Dynamic AtlasES1.0x1.0. Record "1.0x1.0" or "1.0" means that any (at least one: all or any combination) from EA/AtIS eight elements of the Operational stratum that are built from the AtlasSF A1-A8 patterns can be changed by the end user. Herewith only changes that are foreseen by the professional cartographer/cybernetician during development are accessible for the end user. That is why such an atlas remains the atlas of classical type, that is $1.0 \times 1.0=1.0$.

As we say before Web 1.0 EA/AtIS CoFr was received by the abductive reasoning on the example of NAU (Chabanyuk, Dyshlyk, 2014). It was verified on the set of Classical EA/AtIS. The verification set includes our EA/AtIS, National Atlas of Switzerland, National Atlas of Netherlands and some others. All accessible for us EA/AtIS are classical type products: static (Web 1.0) or dynamic (Web $1.0^{2}$ ).

Besides we have used some Information Systems theory facts to make deductive conclusions about correctness of Web 1.0 EA/AtIS CoFr. Than we have used again abductive reasoning to receive Web $1.0^{2}$ EA/AtIS CoFr. Last CoFr was practically verified on AtlasES. We also used deductive reasoning for verification of Web $1.0^{2} \mathrm{EA} / \mathrm{AtIS} \mathrm{CoFr}$ correctness. Now we are investigating Web 2.0 EA/AtIS CoFr architecture. For today we knew few fundamental facts about last $\mathrm{CoFr}$ also as about Web 2.0 ABM CoFr. We are reviewing two of them below.
2.3.1 At the present time in the EA/AtIS projects we use $\beta$ BaseMap1.0 ${ }^{2}$. This framework is realized on the information technologies with the open code: PostgreSQL/PostGIS, MongoDB, GeoServer, Mapnik, Leaflet and so on. Products package of $\beta$ BaseMap1.02 (part of Web $1.0^{2}$ AtPlatform Back-end) has many similarities with OSM platform.

Products package of $\beta$ BaseMap1.02 was created in 2006-2008 as ISGeo Platform with the usage of such commercial software as MapInfo MapXtreme, Oracle etc. ISGeo Platform is used in few commercial projects first of all for Ukrainian telecommunication sector. ISGeo Platform was transferred in open source in last years, when we have developed Web $1.0^{2}$ AtPlatform Back-end.

We need to note that $\beta$ BaseMap $1.0^{2}$ is using most of Web (Mapping) 2.0 technologies, reviewed in (Haklay, et al., 2008). But this rather big set do not give us possibility to use " 2.0 " abbreviation for pointed framework and related with it Web $1.0^{2}$ AtPlatform Back-end. We need to overcome main differences to have $\beta$ BaseMap2.0. These main differences between $\beta$ BaseMap $1.0^{2}$ and OSM are hidden in Processes packages of both systems. OSM platform is used as information tool for BM crowdsourcing. This process can be defined (O'Reilly, 2006) as 'harnessing collective intelligence.' $\beta$ BaseMap $1.0^{2}$ is used mainly for ABM visualization and for the construction of EA/AtIS applications by professionals. To receive $\beta$ BaseMap 2.0 we need to investigate Web 2.0 processes in the EA/AtIS context.

2.3.2 One of the main relations between strata is the relation "meta". For example, National Atlas of Ukraine (NAU or $\omega N A U$ ) is related by "instance-class" relation with metaNAU or $\alpha$ NAU. $\alpha$ NAU is Application stratum element, which can be changed by developers. $\alpha$ NAU is instance of $\beta N A U$ class or $\beta N A U$ is meta- $\alpha$ NAU. Relation "meta" is fundamental method to work with system changes.

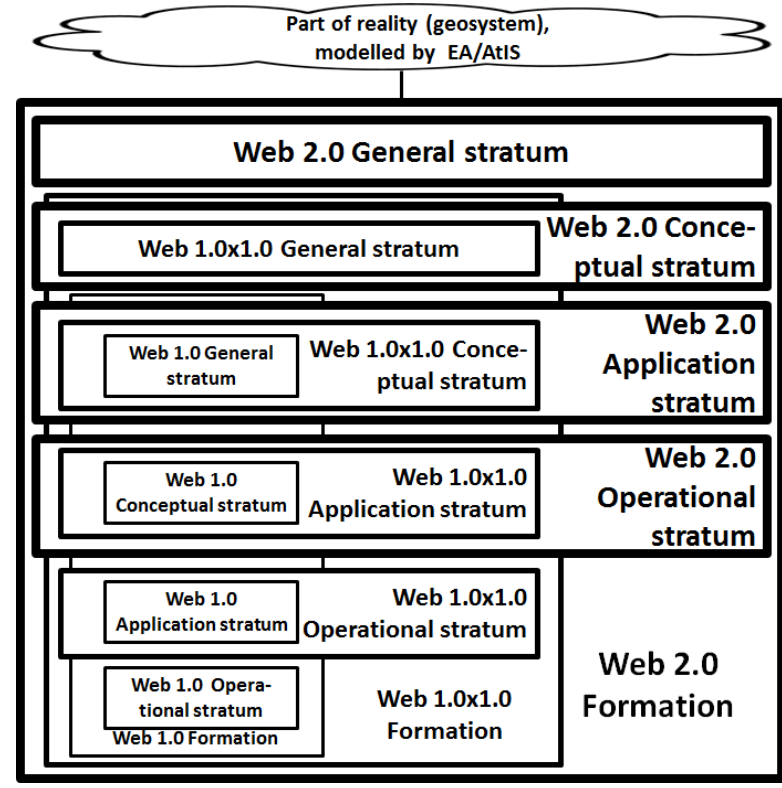

Figure 7. Other view of Formations-Strata projection of EA/AtIS CoFr

As follows from Figure 5 and (Klir, 1985) systems of strata are specific image systems (data, generative, structure, meta-), which can be connected with epistemological levels of systems. So, relation "meta" can be used as powerful investigation method. As we have Web 1.0 and Web $1.0^{2} \mathrm{ABM}$ CoFr with 
"meta" relations we can investigate "increment" between Web 1.0 $0^{2}$ ABM CoFr and Web 2.0 ABM CoFr.

Figure 7 is representing EA/AtIS $\mathrm{CoFr}$ Formations as embedding subsystems: 1) Web 1.0 into Web $1.0^{2}$ and 2) Web $1.0^{2}$ into Web 2.0. Application of Figure 7 to the ABM can help to find elements of highest Web 2.0 stratum by usage of relation "meta".

\section{CONCLUSIONS}

At first glance it may seem that the stated in this work information is unnecessary or very difficult for practical application. Besides we understand that due to volume limitations we could not sufficiently argue the described results. In the following works we are planning to eliminate these defects.

We would like to note that described Web 2.0 ABM CoFr can help to work with BM big data. Some argumentation for it can be found in (Chabanyuk, Dyshlyk, 2015). We also need to note that even Web $1.0^{2} \mathrm{ABM}$ CoFr is including Location Based Services (LBS) as useful practical techniques. We have used LBS in Dynamic AtlasES1.0² prototype (see Figure 3a).

In conclusion we will comment how Web 2.0 ABM Conceptual framework can help to reach motivation aims M1-M5:

1. M1, M3, M4. $\beta$ BaseMap1. $0^{2}$ is constructed on open source software. Thanks to this full control of program and information solutions is reached. In particular we use competitive National topographic map of own making. In case of necessity to use other basemaps of Ukraine (for example, OSM) we solve specific problem of integration of separate elements from BBaseMap2.0 (here is the OSM platform) or $\gamma$ BaseMap1. $0^{2}$ (here are the mechanisms of exchange such as GML). Specific problem is easier to solve than to start from zero.

2. M2. Our Atlas platform has Front-end, architecture of which was developed taking into account obligatory requirement to work on mobile platforms. Thanks to this we can confirm that our solutions are ubiquitous. It must be admitted that we are only at the beginning of the way, whereas much should be done with patterns of the user interface, thematic maps and non-cartographic content for mobile devices. But we hope that new architectural decisions allow doing it in maximum cost-effective way.

3. M5. Working capacity of EA/AtIS of the preceding decade is ensured by means of piecemeal replacement of the broken down elements by new ones built on information technologies with the open code. This issue is one of the most complicated system questions. Largely due to the necessity to maintain the operability of the issued earlier products we had to develop Conceptual frameworks.

\section{REFERENCES}

Andreessen M., 2007. Analyzing the Facebook Platform, three weeks in (accessed 2016-apr-16). http://web.archive.org/web/20071021003047/blog.pmarca.com/ 2007/06/analyzing_the_f.html.

Cartwright W., 2012. Neocartography: Opportunities, Issues And Prospects. South African Journal of Geomatics, Vol. 1, No. 1, pp. 14-31.

Cauvin C., Escobar F., Serradj A., 2010. Thematic Cartography. Volume 3: New Approaches in Thematic Cartography. ISTE-Wiley, 2010.

Chabanyuk V., Dyshlyk O., 2014. Conceptual Framework of the Electronic Version of the National Atlas of Ukraine. Ukrainian Geographical Journal, No. 2, pp. 58-68 (in Ukrainian).

Chabanyuk V., Dyshlyk O., 2015. Atlas Relational Patterns as the Means of Big Data Handling. In: $27^{\text {th }}$ International Cartographic Conference, Rio-de-Janeiro, 17 p.

Decker D., 2000. GIS Data Sources. Wiley.

[Falkenberg E.D., Lindgreen P., Eds., 1989] Falkenberg E.D., Lindgreen P., Eds. Information System Concepts: An In-depth Analysis.- Amsterdam et al., North-Holland, 1989.- 357 p.

Haklay M., Singleton A., Parker C., 2008. Web Mapping 2.0: The Neogeography of the GeoWeb. Geography Compass, Vol. 2, Iss. 6, pp. 2011-2039.

Hart G., Dolbear C., 2013. Linked data: a Geographic Perspective. CRC Press.

Klir G., 1985. Architecture of Systems Problem Solving. Springer.

Kolachny A., 1977. Cartographic Information - A Fundamental Concept and Term in Modern Cartography. Cartographica, Vol. 14, Iss. 1, pp. 39-45.

Kraak M.-J., et al., 2009. The Potential of a National Atlas as Integral Part of the Spatial Data Infrastructure Exemplified by the New Dutch National Atlas. In: SDI Convergence. Research, Emerging Trends, and Critical Assessment. Nederlandse Commissie voor Geodesie Netherlands Geodetic Commission 48, pp. 9-20.

Kraak M.-J., Ormeling F., 2010. Cartography: Visualization of Geospatial Data. Prentice Hall, 3rd Ed.

Kraak M.-J., 2011. Is There a Need for Neo-Cartography? Cartography and Geographic Information Science, Vol. 38, No. 2, pp. 73-78.

O'Reilly T., 2006. Web 2.0 Compact Definition: Trying Again. http://radar.oreilly.com/2006/12/web-20-compact-definitiontryi.html (accessed 2016-mar-06)

Revised May 2016 